\title{
A Complete Analysis of Tolerance of Component in Analog VLSI Circuits Using Sensitivity
}

\author{
J. Vineela, G. Praneetha, R. Harshad, K. Masrunnisa, M. Pruthvi Kumar and T. \\ Sandeep \\ Department of Electronics \& Communication Engineering, AlGIET, Markapur \\ sandeepatism@gmail.com
}

\begin{abstract}
In this paper we focused on practical method to perform tolerance analysis of analog circuits. Any circuit is the combination of passive, controlled and independent sources. The characteristics of analog circuit is totally depends upon the values of components used. So this paper gives detailed analysis of circuit parameter variation. To execute this analysis we use sensitivity method. Using this method we can analyze any analog circuit component tolerance. Tolerance is one of the most important parameter which is considered during the design of analog VLSI circuit. So with the help of sensitivity analysis we can also calculate the component tolerance. We perform sensitivity analysis of various circuit in this paper and show how the value of any component parameter change the circuit behavior. All the equation and model for sensitivity analysis of analog $V L S I$ circuit and simulation are done with the help of MATLAB tool.
\end{abstract}

Keywords: Tolerance, Quality factor, Natural frequency, RLC filter circuit, Integrator circuit, Sallen-key filter

\section{Introduction}

The design of electronic systems/devices must guarantee that all required operating and reliability parameters with minimum manufacturing costs. All types of circuits analog as well as digital, have characteristics depends upon their value of elements. These elements are non-ideal in some ways. The most important characteristics of resistor's is their resistance which is slightly different their nominal value and this characteristics also vary with the time and environment [1].Before designing of any circuit, the designer must know about the sensitivity of various component of circuit that may change with time and change the behavior of circuit. Because the circuit behavior totally depends upon their component values and if component is very critical then the circuit change its behavior with time. And we does not get expected results from our design in future. Analog VLSI circuits also having wide range of application in wireless communication. The basic part of communication device contain analog circuits [2].

Sensitivity analysis of analog circuit provide us the information about various component present in the circuit. The design engineer need to choose as many inexpensive components as possible, by keeping the circuit performance stable, he need to decide which elements are sensitive and how much value they required for the tolerance. With the help of sensitivity analysis we also know about the component characteristics variation in circuit and its effect on performance of system output.

\section{Sensitivity Analysis}

A simple definition of sensitivity is how much specific system behavior/characteristic changes as a individual component value changes [3][4]. The general equation for sensitivity analysis is given below 


$$
S_{x}^{y}=\lim _{\Delta x \rightarrow 0} \frac{\frac{\Delta y}{y}}{\frac{\Delta x}{x}}=\frac{x}{y} \frac{\partial y}{\partial x}
$$

Equation (1) is the general mathematical definition of circuit sensitivity: Where $\mathrm{S}$ represent sensitivity, $\mathrm{X}$ represent changing element/component and $\mathrm{Y}$ is the characteristic of circuit which one want to evaluate as component value is varied. The middle part of this equation shows that the percentage that the dependent variable $\Delta y / y$ changes, relative to the percentage that the independent variable $\Delta \mathrm{x} / \mathrm{x}$ changes. The sensitivity analysis done by using these formulae derived below. Let's take a transfer function $\mathrm{H}(\mathrm{s})$.

$$
\mathrm{H}(s)=\frac{N(s)}{D(s)}
$$

Here $N(s)$ represent the numerator part of transfer function and $D(s)$ represent the denominator part of transfer function. From equation (1) and equation (2), we write a new equation which is same as equation (1). But its variable name are changed to make our calculation easy. In general, the AC-sensitivity is given by the following equation:

$$
\text { Sens }(H(s), W)=\frac{W}{H(s)} \frac{\partial H(s)}{\partial W}
$$

Substituting equation (1) into (2) and applying the chain rule have

$$
\operatorname{Sens}(H(s), W)=W\left(\frac{1}{N(s)} \frac{\partial N(s)}{\partial W}\right)-\left(\frac{1}{D(s)} \frac{\partial D(s)}{\partial W}\right)
$$

Here $\mathrm{W}$ is the component which one want to vary w.r.t. circuit transfer function. By using above equation (3) we can calculate the sensitivity of circuit any circuit.

\subsection{Sensitivity Analysis of Voltage Divider Circuit}

Here we consider voltage divider circuit in Figure 1. And by applying above formulae of sensitivity we derive these equation. This is simplest example we take here. By using this example, we get information about the component of circuit which are sensitive (according to their value).

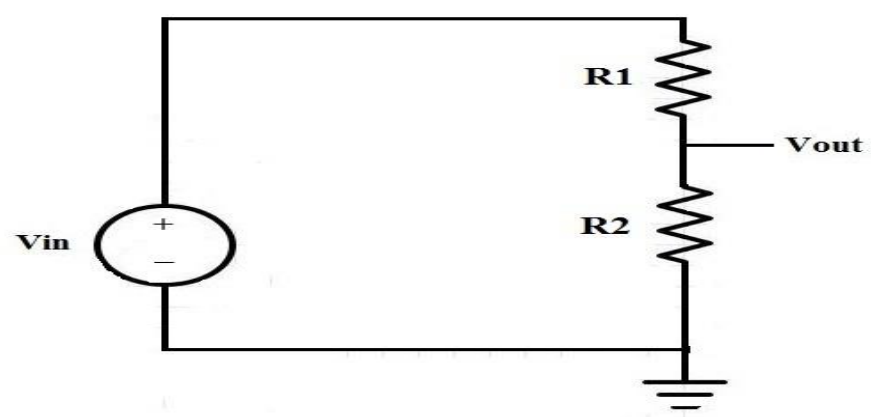

Figure 1. Voltage Divider Circuit

The DC transfer function of is given in equation below

$$
\begin{aligned}
& \mathrm{H}(\mathrm{s})=\frac{\mathrm{V}_{\text {out }}}{V_{\text {in }}}=\frac{R_{2}}{R_{1}+R_{2}}=\frac{\mathrm{N}(s)}{\mathrm{D}(s)} \\
& S_{T_{\text {Dc }}}^{R_{1}}=-\frac{{ }_{R_{1}}}{R_{1}+R_{2}}
\end{aligned}
$$

By using equation (3), we calculate the sensitivities transfer function w.r.t. $R_{1}$ and $R_{2}$.

$$
S_{T_{D C}}^{R_{2}}=\frac{R_{1}}{R_{1}+R_{2}}
$$

In above equation shows that the $\mathrm{DC}$ transfer function is changes w.r.t. $\mathrm{R}_{1}$ and $\mathrm{R}_{2}$.

As shown in equation (4) contain negative sign this implies that if $R_{1}$ increases then cross ponding transfer function decrease. But in case of $R_{2}$ it is opposite that when $R_{2}$ increases then transfer function also increases.In first case, let us assume $R_{1}$ is very large then the equation becomes $-\frac{R_{1}}{R_{1}}=-1$ and $\frac{R_{1}}{R_{1}}=1$. This shows that the transfer function changed by nearly $1 \%$ for $1 \%$ change in either resistor under these condition. 
In second case if $R_{2}$ is very large this results sensitivity equation equal to zero when $R_{1}=0$ and $R_{2}=\infty$. Due to this transfer function changed very small when there is variation in resistor value.

In third case, let us take $R_{1}=R_{2}$ then transfer function becomes 0.5 and sensitivities are -0.5 and 0.5 . Now we expect that the transfer function will changed to $0.5 \%$ for $1 \%$ variation in either resistor. But by increasing $\mathrm{R}_{2}$ to $1 \%$ and transfer function is $1 / 2.01=0.498$ which is reduction of $0.5 \%$. In similar manner we increase $R_{1}$ by $1 \%$ which gives $1.01 / 2.01=0.502$, which is increase of $0.5 \%$. This is sensitivity analysis of very simple circuit which contain only resistor. Now we calculate the sensitivities of circuits which contain resistor, capacitor and inductor.

\section{Analysis of Filter Circuit}

\subsection{Sensitivity Analysis of RLC Filter Circuit}

As we know that all circuit characteristics are function of their element values of circuit. Filter characteristics also dependent on elements values of circuit. Most filter very sensitive to their component values. Sensitivity analysis perform a major role to choose the component values according to their sensitivity [5].

There are many ways to calculate the sensitivity of filters with respect to their component value. One way is that analysis AC transfer behavior of filter with component variation. For simplicity here we write transfer function with $\mathrm{Q}$ (quality factor) and $\omega_{n}$ (natural frequency) [6].

The transfer function of RLC filter circuit is given below

$$
\mathrm{H}(\mathrm{s})=\frac{V_{\text {out }}}{V_{\text {in }}}=\frac{1}{s^{2} L_{1} C_{1}+\frac{L_{1}}{R_{1}}+1}=\frac{N(s)}{D_{(s)}(s)}
$$

Similarly

$$
\begin{aligned}
& \mathrm{H}(\mathrm{s})=\frac{V_{\text {out }}}{V_{\text {in }}}=\frac{1}{\frac{s^{2}}{W_{n}{ }^{2}}+\frac{s}{W_{n} Q}+1}=\frac{\mathrm{N}(s)}{\mathrm{D}(s)} \\
& \omega_{n}=\frac{1}{\sqrt{L_{1} C_{1}}} \\
& \mathrm{Q}=R_{1} \sqrt{\frac{C_{1}}{L_{1}}}
\end{aligned}
$$

Using nominal values of RLC circuit the $\mathrm{Q}=0.707$.by using general equation of sensitivity the sensitivities of $\omega_{n}$ and $\mathrm{Q}$ are given below.

Sensitivity using $Q$ (quality factor)

$$
\begin{aligned}
& S_{C_{1}}^{Q}=-S_{L_{1}}^{Q}=\frac{1}{2} \\
& S_{R_{1}}^{Q}=1
\end{aligned}
$$

And sensitivity using $\omega_{n}$ (natural frequency)

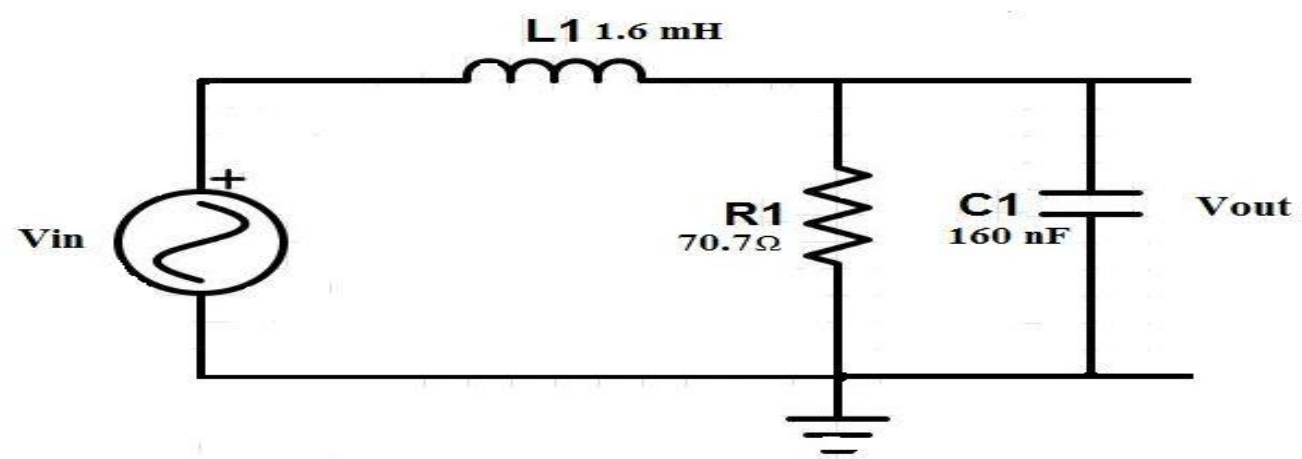

Figure 2. Circuit Diagram of RLC Filter Circuit 


$$
\begin{aligned}
& S_{C_{1}}^{\omega_{n}}=-S_{L_{1}}^{\omega_{n}}=-\frac{1}{2} \\
& S_{R_{1}}^{\omega_{n_{n}}}=0
\end{aligned}
$$

As we saw that resistor value does not depends upon natural frequency in above equation.

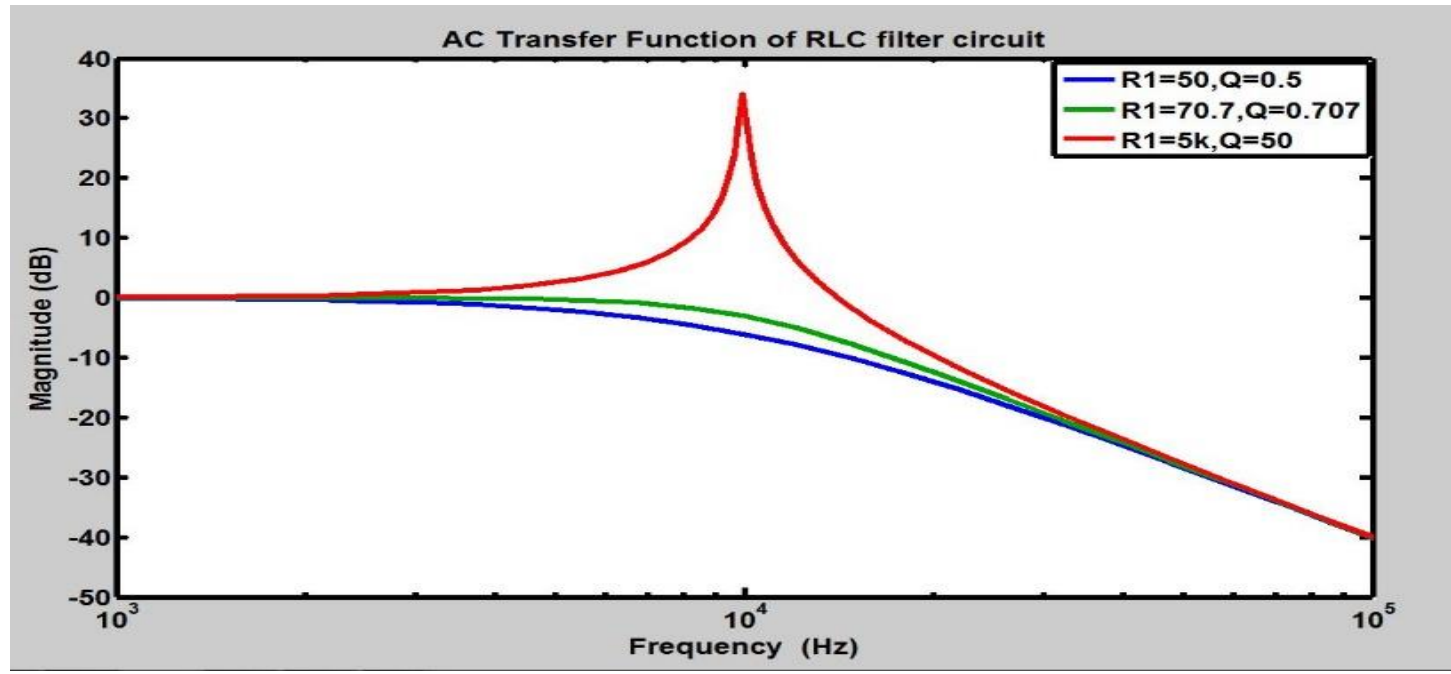

Figure 3. Variation of $R_{1}$ w.r.t $Q$

Figure 3 shows the variation of $R_{1}$ w.r.t quality factor. Because it is most sensitive component in this circuit (in term of quality factor sensitivity).By using equation (3) and equation (6) we derive sensitivities of components which are given below.

Here $\mathrm{W}$ is the component which we want to vary. So $\mathrm{W}=\mathrm{L}_{1}$

$$
\begin{aligned}
& =\mathrm{L} 1\left[-\frac{1}{s^{2} L_{1} C_{1}+s s_{1}} \frac{\partial\left(s^{2} L_{1} C_{1}+s \frac{L_{1}}{R_{1}}+1\right)}{\partial L 1}\right] \\
& =\mathrm{L} 1\left[-\frac{\left(s^{2} C_{1}+\frac{s_{1}}{R_{1}}\right)}{s^{2} L_{1} C_{1}+s \frac{L_{1}}{R_{1}}+1}\right] \\
& \mathrm{S}_{L_{1}}^{\mathrm{H}(s)}=\left[-\frac{\left(s^{2} L_{1} C_{1}+s \frac{L_{1}}{R_{1}}\right)}{s^{2} L_{1} C_{1}+s_{1} \frac{L_{1}}{R_{1}}+1}\right]
\end{aligned}
$$

Similarly we calculate for $R_{1}$ and $C_{1}$

$$
\begin{aligned}
& S_{R_{1}}^{H(s))}=\left[-\frac{\left(s \frac{L_{1}}{R_{1}}\right)}{s^{2} L_{1} C_{1}+s_{R_{1}}}\right] \\
& S_{C_{1}}^{H(s)}=\left[-\frac{\left(s^{2} L_{1} C_{1}\right)}{s^{2} L_{1} C_{1}+s_{1} L_{R_{1}}+1}\right]
\end{aligned}
$$

Here we plot sensitivity of passive elements with nominal values as well as some tolerance provided to nominal value and by using this we get the information related to sensitivity of elements in RLC circuit.

With the help of sensitivity analysis one also know about the tolerance specified at the time of design in particular element. Designer can provide cheap elements which does not affect circuit characteristics. By using equation (10), (11) and (12).

We analyzed the sensitivity of RLC filter circuit. 


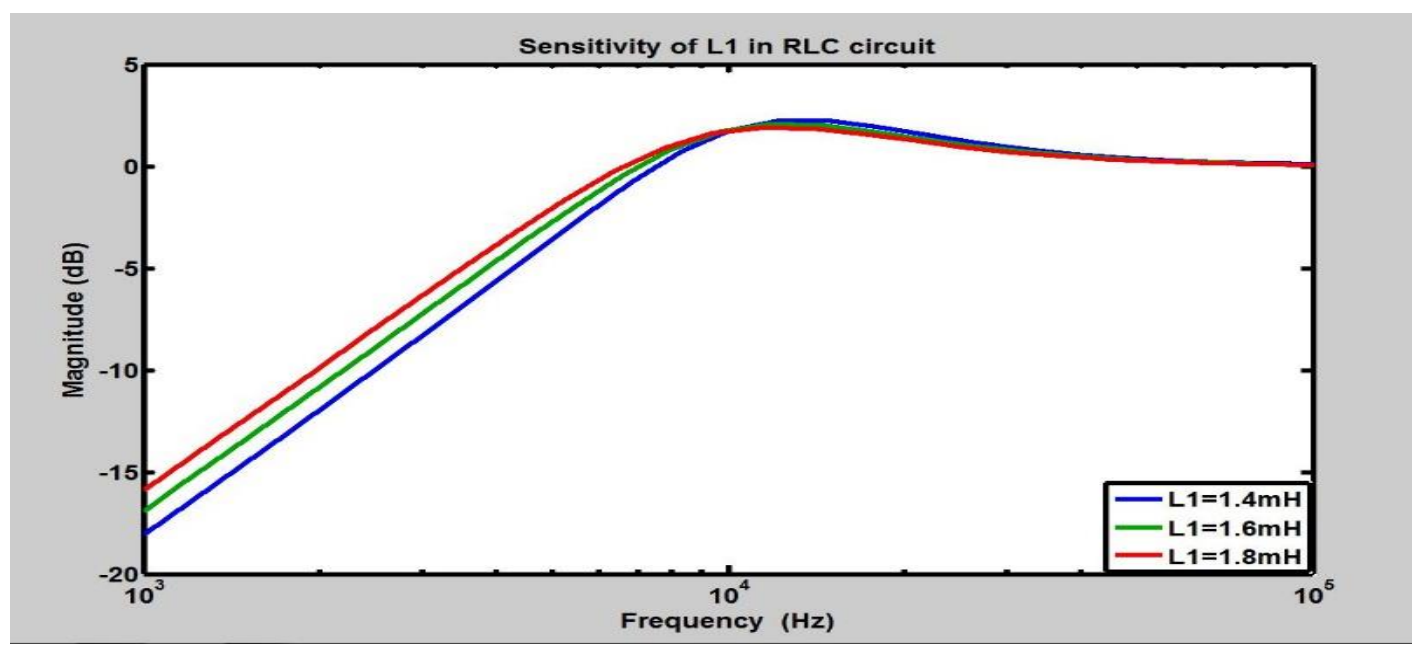

Figure 4. Sensitivity of Inductor in RLC Circuit

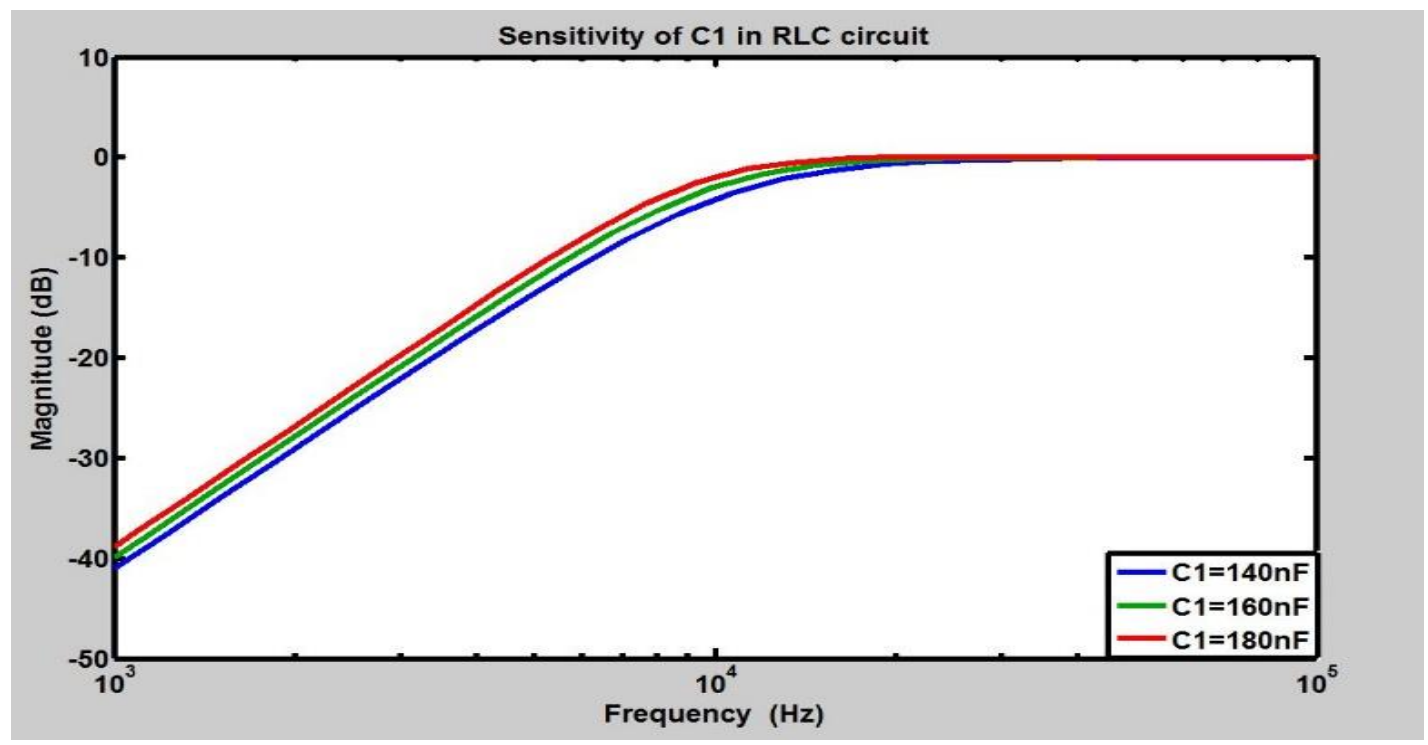

Figure 5. Sensitivity of Capacitor in RLC Circuit

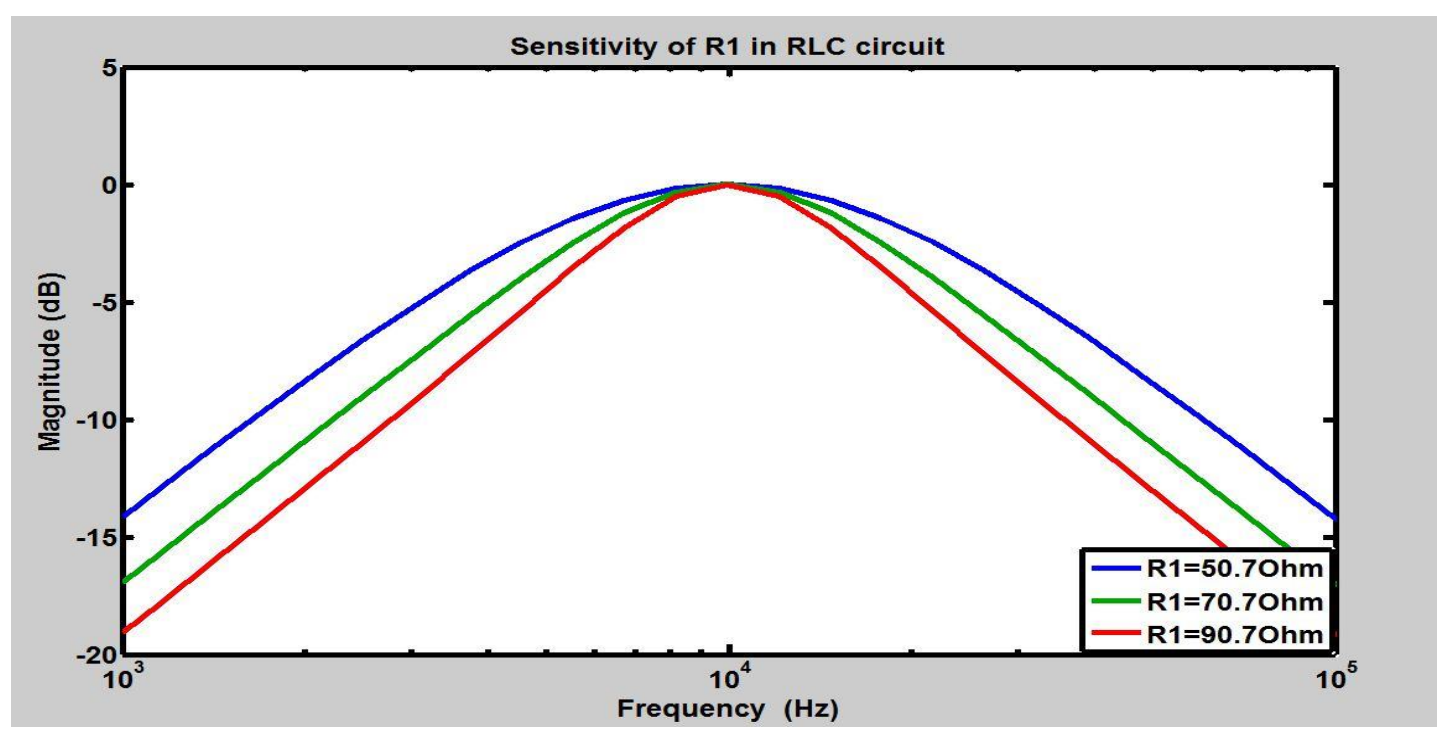

Figure 6. Sensitivity of Resistor in RLC Circuit 


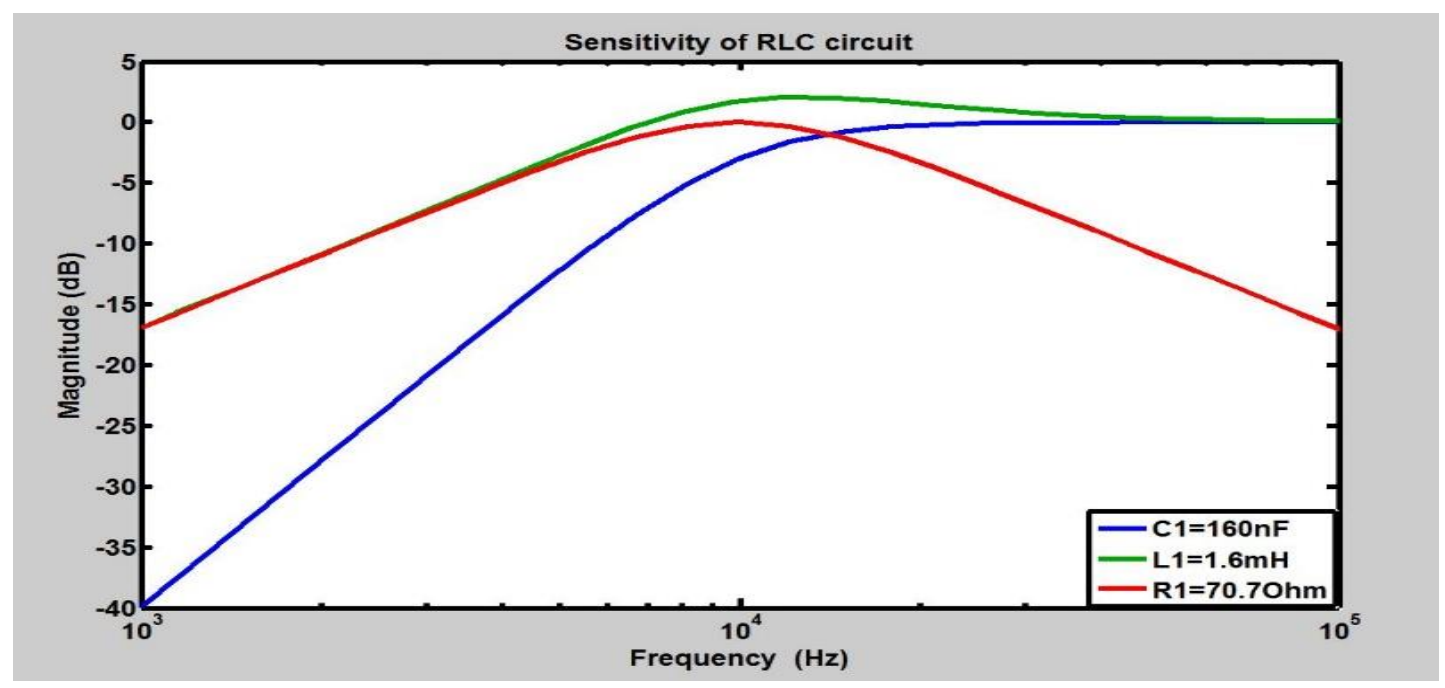

Figure 7. Comparison of Sensitivities of Passive Elements in RLC Circuit

In Figure 4, 5 and 6 we plot sensitivity analysis using nominal values of component and also provide some tolerance form their nominal value. Figure 7 shows the passive component sensitivities of RLC circuit, using this information we can easily analyze the most sensitive and less sensitive component present in circuit.

\section{Analysis of Integrator Circuit}

In this part of paper we describe the sensitivity analysis of integrator circuit which is basic element of various circuit. Figure 8 show the circuit diagram of integrator circuit. Here the transfer function of integrator circuit is given [7].

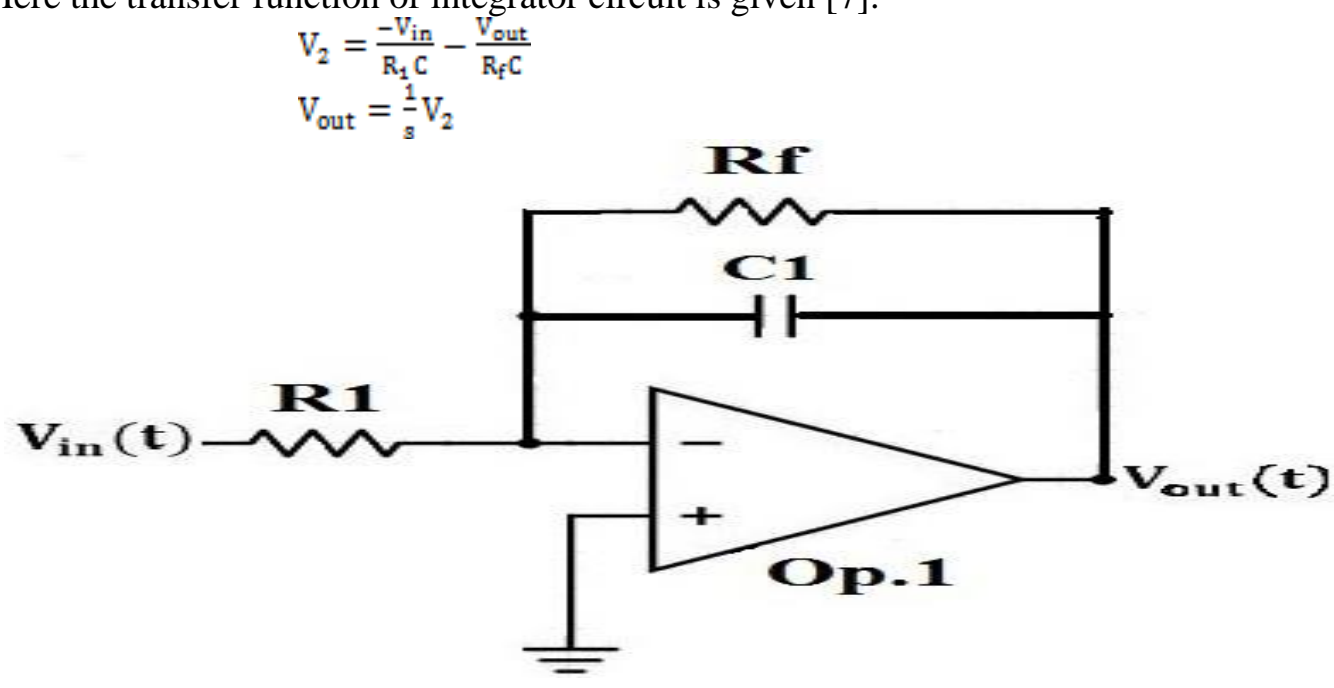

Figure 8. Circuit Diagram of Integrator Circuit

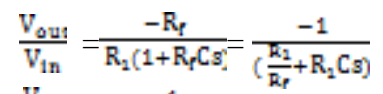

$$
\begin{aligned}
& \frac{V_{\text {oun }}}{V_{\text {ln }}}=\frac{-1}{\left(\frac{k_{1}}{R_{\mathrm{r}}}+R_{2} \mathrm{C}(s)\right.}
\end{aligned}
$$

Sensitivity analysis of integrator is given below

$$
\mathrm{S}_{\mathrm{R}_{f}^{(s)}}^{\mathrm{H}(s)}=1-\frac{R_{\mathrm{R}} \mathrm{Cs}}{1+\mathrm{R}_{\mathrm{f}} \mathrm{Cs}}
$$

Similarly for capacitor $\mathrm{C}$

$$
\mathrm{S}_{\mathrm{C}}^{\mathrm{H}(s)}=\frac{-\mathrm{R}_{\mathrm{p}} \mathrm{Cs}}{1+\mathrm{R}_{\mathrm{p}} \mathrm{Cs}}
$$


Here $\mathrm{R}_{\mathrm{f}}=100 \mathrm{k}$ and $\mathrm{C}=0.02 \mu \mathrm{F}$

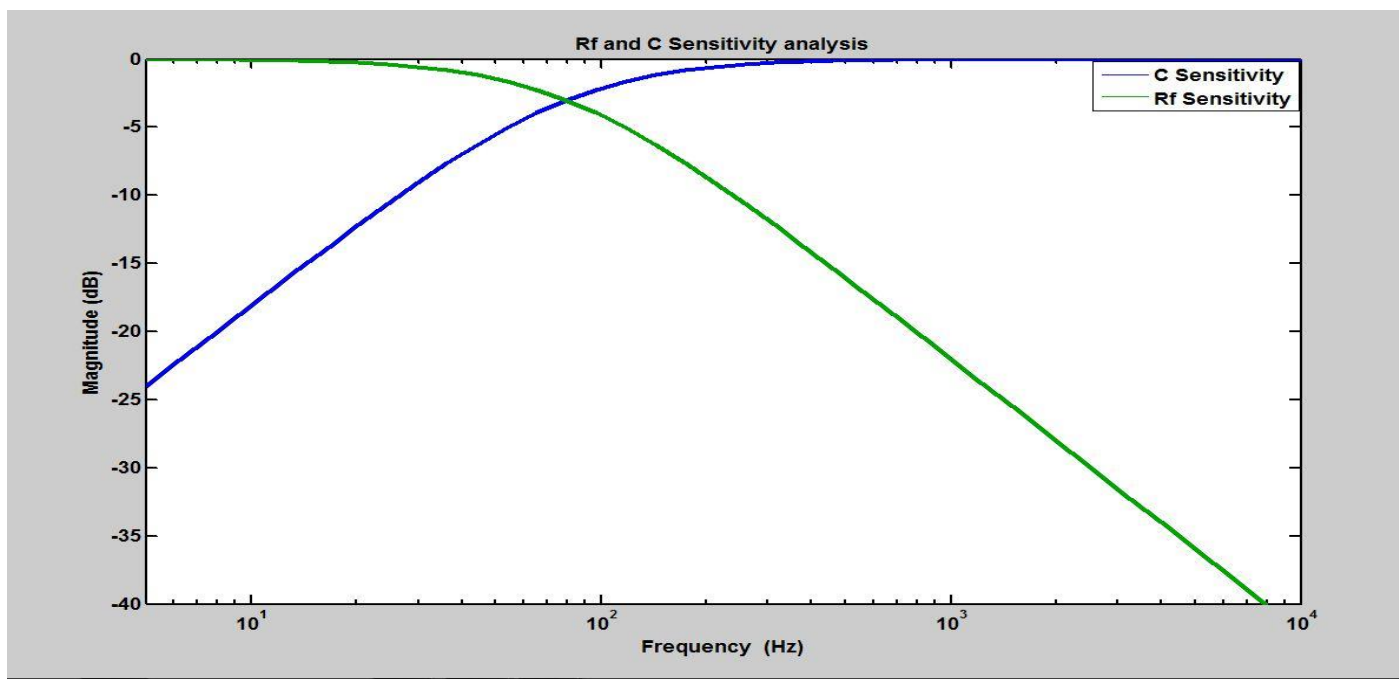

Figure 9. Sensitivity Analysis of Components in Integrator Circuit

\section{Analysis of Active Filter Circuit}

Sallen-key filter one of the most common filter [8][9]. The transfer function of this filter is

$$
\frac{V_{\text {out }}}{V_{\text {in }}}=\frac{K}{\mathbb{R}^{2}\left(R_{1} R_{3} C_{2} C_{4}\right)+\&\left(R_{1} C_{2}+R_{3} C_{4}+R_{1} C_{4}-K R_{1} C_{2}\right)+1}
$$

Where $\mathrm{K}=1+\frac{\mathbb{R}_{b}}{\mathrm{R}_{a}}$

$$
\begin{aligned}
& \omega_{n}=\frac{1}{\sqrt{R_{1} R_{3} C_{2} C_{4}}} \\
& \frac{1}{Q}=\sqrt{\frac{R_{3} C_{4}}{R_{1} C_{2}}}+\sqrt{\frac{R_{1} C_{4}}{R_{3} C_{2}}}++(1-K) \sqrt{\frac{R_{1} C_{2}}{R_{3} C_{4}}}
\end{aligned}
$$

Sensitivity of K w.r.t Q is

$$
\begin{aligned}
& S_{K}^{Q}=Q K \sqrt{\frac{R_{1} C_{2}}{R_{3} C_{4}}} \\
& S_{R_{a}}^{Q}=-S_{R_{b}}^{Q}=-Q(K-1) \sqrt{\frac{R_{1} C_{2}}{R_{3} C_{4}}}=-Q \frac{R_{b}}{R_{a}} \sqrt{\frac{R_{1} C_{2}}{R_{3} C_{4}}}
\end{aligned}
$$

Sensitivity of active filter component w.r.t $\omega_{n}$ is

$$
\begin{aligned}
& S_{R_{1}}^{\omega_{n_{n}}}=S_{R_{3}}^{\omega_{n_{n}}}=S_{C_{2}}^{\omega_{n_{n}}}=S_{C_{4}}^{\omega_{n_{n}}}=-\frac{1}{2} \\
& S_{K}^{\omega_{n}}=S_{R_{a}}^{\omega_{n_{n}}}=S_{R_{b}}^{\omega_{n_{n}}}=0
\end{aligned}
$$

From above equation it is clear that natural frequency sensitivities of passive filter is $\pm 0.5 \%$ or Zero. But Q sensitivities are little complex.

The nominal values of component in Sallen key filter are $\mathrm{R}=10 \mathrm{~K} \Omega$ and $\mathrm{C}=16 \mathrm{nF}$ [10]. 


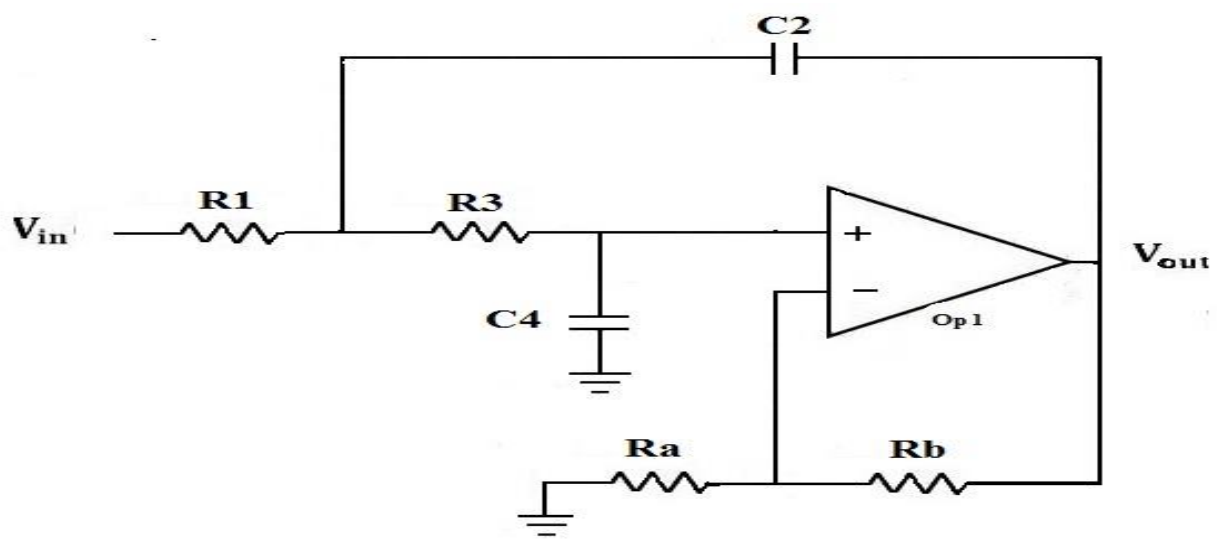

Figure 10. Sallen-Key Filter Circuit Diagram

The Sallen key filter in figure is $1 \mathrm{kHz}$ (low pass filter) with $\mathrm{Q}=1$. In this filter we vary the value of $R_{b}$ by keeping natural frequency constant. We vary the value of $R_{b}$ from $1 \mathrm{~K} \Omega$ to $19.9 \mathrm{~K} \Omega$. At $R_{b}=20 \mathrm{~K} \Omega$ the $Q$ goes to very high so we avoided it. As we know that $R_{b}$ is main gain determine component in filter changing with $\mathrm{Q}$ in Figure.10.Figure 11 shows the variation of different values for $\mathrm{R}_{\mathrm{b}}$. All simulation are done with the help of MATLAB software [11].

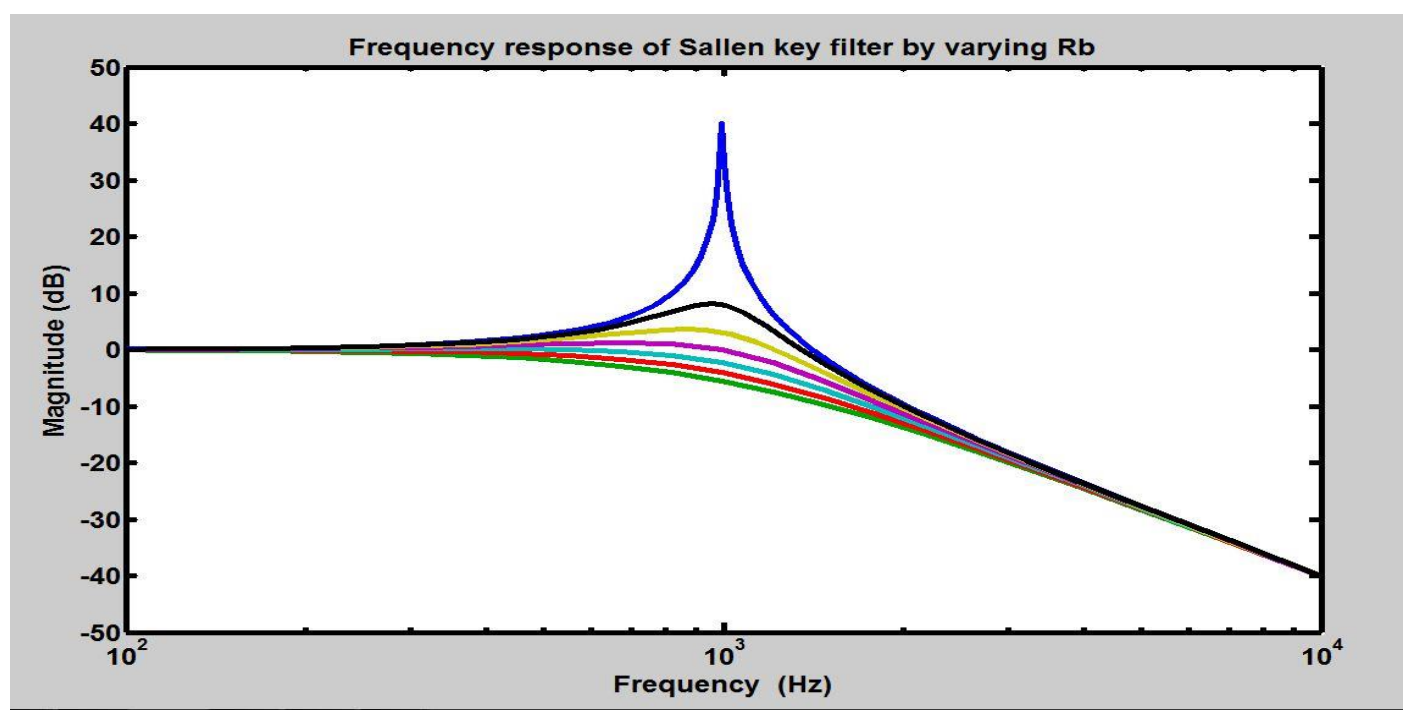

Figure 11.i.e Response of Sallen-Key Filter by Varying $R_{b}$ From $1 \mathrm{~K} \Omega$ to $19.9 \mathrm{~K} \Omega$

\section{Result Analysis}

By performing this analysis, we learn various aspects of analog VLSI circuits. That is calculation of $\omega_{n}$ and $\mathrm{Q}$ sensitivities are very easy task. This analysis also provide us a valuable information of component sensitivity, by using that we can set the tolerance of component in the circuit. For example, if the component is very sensitive then we have to specify the tolerance of that component very close to nominal value. This analysis reduce the designing cost of analog VLSI circuits and also provide the information about tolerance of component, which made our design cheaper and give expected results. Due to this we also increase the aging factor of the device. 


\section{Conclusion}

In this paper we analyze various analog circuits and perform their tolerance analysis using MATLAB tool. The analysis provide us various information about the component present in the circuit and how they change the characteristics of the circuit. This work helps the design engineer to reduce the cost of analog VLSI circuits and also increase the life span of the device. This paper describe the detail method of tolerance analysis of analog VLSI circuit with the help of sensitivity analysis.

\section{Acknowledgments}

The authors express deep sense of gratitude to Mr. Shamsheer Ali Baig, Honorable chairman of A1 Global Institute of Engineering \& Technology for his vision and encouragement towards research and development and providing various facilities to do this research. Also the authors would like to express their gratitude to Dr. Ch. Sreedhar, Principal and Mr. K.V.V Satayanarayana, Vice principal (HOD ECE) for their support. Authors would like to thank all the Electronics and Communication staff member for their support.

\section{References}

[1] Y. Lu and R. Dandapani, "Hard Fault Diagnosis in Analog Circuits Using Sensitivity Analysis", Proc. of the IEEE VLSI Test Symp., (1993), pp. 225-229.

[2] S. Thakur, "A Complete Analysis of Channel Estimation and Peak to Average Power Ratio in Wireless Communication Using Discrete Fourier Transform", (2016).

[3] A. C. Sanabria-Borbon and E. Tlelo-Cuautle, "Symbolic sensitivity analysis in the sizing of analog integrated circuits", Computing Science and Automatic Control, (2013).

[4] R.P. Sallen, and E.L. Key, "A Practical Method of Designing Active Filters", IRE Transactions on Circuit Theory, March, vol. CT-2, (1955), pp.74-85.

[5] L.P. Huelsman, and P.E. Allen, "Introduction to the Theory and Design of Active Filters", McGrawHill, New York, (1980).

[6] L. Milor and V. Visvanathan, "Detection of Catastrophic Faults in Analog Integrated Circuits", IEEE Trans. on Computer-Aided Design, vol. 8, (1989), pp. 114-130.

[7] N.B. Hamida and B. Kaminska, "Multiple Fault Analog Circuit Testing by Sensitivity Analysis", J. Electronic Testing: Theory and Applications, vol. 4, (1993), pp. 331-343.

[8] B. Kaminska, K. Arabi, I. Bell, P. Goteti, J.L. Heurtas, B. Kim, A. Rueda and M. Soma, "Analog and Mixed-Signal Benchmark Circuits First Release", IEEE International Test Conference, Washington DC, (1997).

[9] S.-J. Tsai, “Test Vector Generation for Linear Analog Devices”, Proc. of the IEEE Int'l. Test Conf., Oct., pp. 592-597, (1991).

[10] N. Balabanian, T.A. Bickart and S. Seshu, "Electrical Network Theory", John Wiley \& Sons, Inc., chap. 9, (1969), pp. 646-647.

[11] The Math Works Inc. MATLAB User's Guide, (2009). 
International Journal of Hybrid Information Technology Vol. 9, No.7 (2016) 